\title{
Hans Joas
}

\section{Pragmatismus und Historismus}

\author{
Meads Philosophie der Zeit und die Logik der \\ Geschichtsschreibung
}

\begin{abstract}
This article explores the similarities between American pragmatism and (mostly German) historicism in the nineteenth century - similarities that were often ignored because of cultural differences between the U. S. and Germany and a different status of the natural sciences or the humanities in the two cultures. The main claim of this text is that American pragmatism developed ideas that allow us to overcome the dichotomy between objectivism and relativism in historiography. Joas identifies conceptual tools in the works of Josiah Royce, Mead, and Dewey that can account both for the intersubjective and the temporal nature of human experience and the processes of the formation of ideals. By bringing Ernst Troeltsch, the most sophisticated thinker from the historicist tradition, into the picture, Joas demonstrates that in the 1920s one could almost speak of the beginning of a process of convergence between Mead's "temporalized pragmatism” and Troeltsch's "existential historicism." For contingent reasons this convergence never took place, but remains a challenge to which this paper responds.
\end{abstract}

Keywords: pragmatism, historicism, history, time, temporality, ideals, relativism, objectivity

DOI 10.1515/dzph-2015-0001

Im Jahr 1988 veröffentlichte der angesehene Chicagoer Historiker Peter Novick ein meisterhaftes Buch zur Geschichte der amerikanischen Geschichtswissenschaft $^{1}$. Dieses Buch bietet eine Fülle von Material und Interpretationen zur Entwicklung der historischen Profession in den USA, zu ihren bevorzugten Themengebieten und blinden Flecken und $\mathrm{zu}$ den politischen Voreingenommenheiten und Einseitigkeiten einer angeblich unpolitischen wissenschaftlichen Disziplin. In erkenntnistheoretischer Hinsicht - oder, besser gesagt, in Hinsicht auf die epi-

1 Novick (1988).

Prof. Dr. Dr. h.c. Hans Joas: Ernst-Troeltsch-Honorarprofessor, Theologische Fakultät, HumboldtUniversität zu Berlin, Unter den Linden 6,10099 Berlin; hans.joas@hu-berlin.de 
stemologischen Überlegungen, die von den Historikern selbst angestellt wurden - arbeitet Novick ein ständiges Schwanken zwischen dem Ideal der Objektivität dem „edlen Traum“, von dem Charles Beard 1935 gesprochen hatte - und dem, was oft als „Relativismus“ bezeichnet wird, heraus. Mit dieser Strukturierung des Feldes epistemologischer Alternativen waren allerdings nicht alle Rezensenten von Novicks Buch glücklich ${ }^{2}$. Sie wiesen darauf hin, daß kaum jemals ein Gelehrter sich selbst wirklich als Relativisten bezeichnet hat. Meistens handelt es sich nicht um eine Selbst-, sondern eine Fremdbezeichnung, ein Etikett, das von den Verteidigern des jeweiligen Mainstreams gerne gegen ihre Gegner verwendet wird, ganz unabhängig davon, wie deren Selbstverständnis beschaffen ist. Aber die Gefahren des dichotomischen Denkschemas „Objektivismus versus Relativismus“ gehen über die verzerrte Wahrnehmung von Forschungsansätzen hinaus. Die Dichotomie selbst wird nämlich nur allzu leicht verdinglicht. Sie erscheint dann als erschöpfende Charakterisierung der Denkmöglichkeiten, das in ihr enthaltene Dilemma damit als unüberwindbar. Einer der Rezensenten, der Harvard-Historiker James Kloppenberg, wandte deshalb ein, daß Novick durch seine Vorgehensweise weder „unser Verständnis der substantiellen Fragen im Zusammenhang des Problems der Objektivität“"3 erweitert noch genügend Aufmerksamkeit der Geschichte von Versuchen gewidmet habe, einen Weg aus diesem Dilemma heraus zu finden. Zwar trifft es zu, daß sich die differenziertesten Versuche in dieser Richtung nicht so sehr in den Schriften praktizierender Historiker finden, sondern eher in anderen Disziplinen wie der Philosophie, Soziologie und Theologie. Aber es bleibt doch eine auffallende Lücke in Novicks Buch, daß er auf solche Versuche nicht recht eingeht, selbst dann, wenn sie typisch amerikanischen intellektuellen Traditionen entstammen.

Mir geht es mit diesen Bemerkungen und in diesem Beitrag natürlich nicht um eine detaillierte Kritik an Novicks brillantem Buch. Mir geht es vielmehr um die These, daß der amerikanische Pragmatismus Ideen entwickelt hat, die in grundlegender Weise über den Dualismus von Objektivismus und Relativismus hinausführen, „beyond objectivism and relativism“ liegen, wie der bedeutende Neopragmatist Richard Bernstein eines seiner Bücher ${ }^{4}$ genannt hat. Diese Ideen finden sich vor allem, chronologisch geordnet, im letzten großen Werk von Josiah Royce, dem Buch The Problem of Christianity von 1913, in George Herbert Meads Philosophy of the Present, die 1932 ein Jahr nach seinem Tod in unvollendeter

2 Vgl. Kloppenberg (1989), American Historical Review (1991) (Buchsymposium) u. Haskell (1998).

3 Kloppenberg (1989), 1015.

4 Bernstein (1983). 
Form erschien, und in John Deweys Logik von 1938. Neben einer Erinnerung an diese Ideen geht es mir vor allem darum, diese intellektuelle Tradition auf eine andere $\mathrm{zu}$ beziehen, die auf den ersten Blick denkbar weit von ihr entfernt und ganz anders beschaffen erscheinen mag: die des (vornehmlich) deutschen Historismus. Ich werde behaupten, daß diese beiden Denkschulen, Pragmatismus und Historismus, von ihrem jeweiligen Beginn an beträchtliche Ähnlichkeiten miteinander aufwiesen und im Lauf der Zeit einander sogar immer ähnlicher wurden. Das Ausmaß dieser Annäherung war so groß, daß man von einer sich abzeichnenden Konvergenz in den 1920er Jahren sprechen kann, wenngleich - aus historisch kontingenten Gründen - durch den relativen Abbruch beider Traditionen danach die Konvergenz nie zustande kam. Den genauen Konvergenzpunkt zu bezeichnen - ohne dabei die verbleibenden Differenzen der Schulen zu ignorieren - stellt zugleich eine substantielle These zur Logik historischer Forschung und insbesondere der Wert-Dimension aller Geschichtsschreibung dar.

Aus drei Gründen sind die tiefen Gemeinsamkeiten von (amerikanischem) Pragmatismus und (deutschem) Historismus bei oberflächlicher Betrachtung nicht wahrzunehmen. Zum einen sind die disziplinären Schwerpunkte der beiden Denkschulen unterschiedlich. Der Pragmatismus entwickelte sich aus den Naturwissenschaften heraus. Sowohl Charles Sanders Peirce wie William James waren praktizierende Naturwissenschaftler. Für beide gilt, daß ihre philosophischen Schriften ohne den Bezug zu der wissenschaftlichen Arbeit, aus der sie entstanden, gar nicht angemessen zu verstehen sind. Der empirische Hintergrund von John Dewey und George Herbert Mead war zwar nicht in den „harten“ Naturwissenschaften, sondern in der Psychologie - aber in dieser damals jungen empirischen Disziplin war ihr Hauptbestreben das einer „Naturalisierung“ dessen, was die philosophische Tradition als mentale Phänomene verbucht hatte. Die Tatsache, daß es ihnen gerade um eine nicht-reduktionistische Naturalisierung ging, ändert daran nichts.

Der deutsche Historismus dagegen entstammte den im neunzehnten Jahrhundert an den Universitäten des deutschen Sprachraums so großartig aufblühenden, international führenden historischen Geistes- und Staatswissenschaften. Seine Grundlage und sein Anwendungsfeld hatte er in Rechts- und Wirtschaftsgeschichte, der historisch-kritischen Bibelwissenschaft, Christentums- und Religionsgeschichte, der Geschichte der Philosophie und der Literaturen. Hinter diesen unterschiedlichen disziplinären Schwerpunkten steht eine kulturelle Differenz zwischen Deutschland und den USA, die eine Gemeinsamkeit der beiden Strömungen verbirgt. Es handelte sich nämlich jeweils um das in der jeweiligen Kultur prestigereichste und einflußstärkste akademische Feld. Zudem standen beide Strömungen in einer komplexen kritischen, aber keineswegs destruktiven Beziehung zu den religiösen Traditionen des Christentums. In 
dieser kulturellen Differenz zwischen einem stärker naturwissenschaftsbezogenen Fortschrittsoptimismus in den USA und einem stärker um nationale Identität ringenden Geschichtsinteresse in Deutschland kann man einen zweiten Grund für die Verdeckung der Gemeinsamkeiten sehen.

Ein dritter Grund, der vielleicht aber mehr einen bloßen Ausdruck der genannten Differenz darstellt, liegt in den unterschiedlichen Konnotationen der beiden Schul-Namen selbst. In Deutschland war der Begriff Pragmatismus bei fast allen Philosophen - von Husserl bis Horkheimer - äußerst negativ besetzt. Man assoziierte mit ihm eine oberflächliche oder zynische Reduktion der heiligen philosophischen Wahrheitssuche auf alltägliche oder kapitalismusdienliche Nützlichkeitserwägungen. ${ }^{5}$ Umgekehrt wurde die Bezeichnung „Historismus“ in den USA meist nicht im Sinne eines radikalen Bewußtseins von der Geschichtlichkeit aller menschlichen Phänomene verwendet, sondern als Etikett für die naive Vorstellung, daß Historiker ihre Behauptungen durch eine Überprüfung an der Vergangenheit an sich überprüfen könnten. Fast grotesk wurde das Mißverständnis des „Historismus“, als Karl Raimund Popper in seiner polemischen Schrift von 1960 Das Elend des Historizismus den Begriff zur Kennzeichnung einer vulgären teleologischen Geschichtsphilosophie verwendete, die sich auf Hegel oder Marx berief.

Wegen des unterschiedlichen disziplinären Hintergrunds von Pragmatismus und Historismus unterschieden sich auch die bevorzugten Themenfelder der beiden Denkschulen beträchtlich. Die Pragmatisten zeigten lange Zeit kein starkes Interesse an den historischen Wissenschaften. In den Worten von Peter Novick zollten Peirce, James und Dewey „historischen Fragen praktisch keine Aufmerksamkeit “ ${ }^{6}$. Zwar studierten einige bedeutende amerikanische Historiker (wie James Harvey Robinson) und historisch orientierte Soziologen (wie W. E. B. Du Bois) bei William James oder zeigten (wie Charles Beard) Sympathie für das Denken John Deweys; dennoch läßt sich sagen, „daß es wenig Grund gibt, anzunehmen, daß der Pragmatismus vor der Zwischenkriegszeit irgendeine substantielle Verbindung mit dem historischen Denken aufwies“"7. In Deutschland wiederum sahen sich die „historistischen Historiker“ selbst als in einer ständigen Abwehrschlacht befindlich gegen materialistische und naturalistische Feinde. $\mathrm{Zu}$ diesen gehörten für sie nicht nur die Marxisten, sondern alle, die ihre Inspiration aus den Naturwissenschaften bezogen und etwa eine milieudeterministische Kulturgeschichte vertraten. Diese Abwehrhaltung führte bei den meisten dazu,

5 Vgl. Joas (1992).

6 Novick (1988), 151.

7 Etwas optimistischer ist die Einschätzung bei Kloppenberg (2004). 
daß sie sich auch nicht für die doch unbezweifelbar biologischen Grundlagen der für den Historismus konstitutiven Auffassungen über menschliches Handeln interessierten. Eine Ausnahme stellte Wilhelm Dilthey dar, der sich vor allem in seiner mittleren Phase genau darum bemühte. ${ }^{8}$ Die eigentlich radikale Änderung aber trat erst mit der sogenannten „Philosophischen Anthropologie“ Max Schelers und Helmuth Plessners ein. ${ }^{9}$

Worin aber bestanden die hinter den oberflächlichen Differenzen liegenden Gemeinsamkeiten? Am raschesten lassen sich diese identifizieren, wenn wir den Anti-Cartesianismus beider Strömungen in den Blick nehmen. Für Peirce war bekanntlich die Zurückweisung des Prinzips methodologischen oder radikalen Zweifels zentral und seine Ersetzung durch die Vorstellung, daß das Denken nicht auf dem Wege künstlicher Infragestellungen alltäglicher Selbstverständlichkeiten durch Philosophen voranschreitet, sondern nur durch realen, situierten Zweifel, durch die echte Infragestellung bisheriger Selbstverständlichkeiten, weil diese zu Schwierigkeiten im Handeln geführt haben. Die Lösung solcher realen Handlungsprobleme, so der Grundgedanke des Pragmatismus, liegt immer in kreativen Akten, die selbst wieder im Erfolgsfall zu neuen Handlungsgewohnheiten werden und das Problemlösungsrepertoire der Handelnden erweitern. Dabei setzt jede schöpferische Problemlösung voraus, nicht alles gleichzeitig in Frage zu stellen, sondern den Kontext als solchen als unproblematisierten Hintergrund dahingestellt sein zu lassen. George Herbert Mead benutzte für diese unproblematisierte Welt den Begriff der Welt „that is taken for granted“, ein Begriff, der dem Lebenswelt-Begriff des späten Husserl ähnelt, ihm allerdings zeitlich vorausgeht. ${ }^{10}$

Auf der Seite der Historisten entsprechen die Überlegungen zu unserem Verstehen von Texten den pragmatistischen Überlegungen zur Lösung von Handlungsproblemen. Von Schleiermacher und anderen ausgehend, vor allem aber von Dilthey minutiös durchdacht, wird die Begegnung des Lesers mit dem Text als eine nie voraussetzungslose erläutert. Wenn wir einem Text gegenübertreten, tun wir dies immer schon auf der Grundlage einer Fülle uns selbst nicht präsenter Annahmen über die Welt, uns selbst und den Text, mit dem wir uns auseinandersetzen. Im Umgang mit dem Text stoßen wir auf Äußerungen des Verfassers, die diese mitgebrachten Auffassungen herausfordern. Dies können direkt unsere Vorstellungen bestreitende Thesen, aber auch stillschweigende, von unseren Vorstellungen abweichende Unterstellungen des Autors sein. Wir stoßen vor allem

8 Vgl. Dilthey (1924b); dies wird von Jung (1996) hervorgehoben.

9 Vgl. Honneth/Joas (1980).

10 Vgl. Mead (1938). 
aber auch auf Stellen, die uns dunkel und unverständlich scheinen. Um diese aufzuhellen, begeben wir uns tiefer in den Text hinein, lassen uns von der Vorstellung leiten, daß sich ihr Sinn aus dem Sinn anderer Stellen gewinnen läßt, und werden so in ein Hin und Her zwischen verbessertem Textverständnis und wachsendem Selbstverständnis hineingezogen. Dieses Hin und Her wird oft als „hermeneutischer Zirkel“ bezeichnet, obwohl es sich gerade nicht um einen Zirkel handelt, bei dem wir ohne Gewinn an Einsicht zum Ausgangspunkt der Überlegungen bloß zurückkehrten.

Mit dieser Betonung der Situiertheit allen Erkennens sowohl bei der Lösung technisch-naturwissenschaftlicher wie interpretativ-geisteswissenschaftlicher Fragen stellt sich auch ein fundamentaler Zweifel an den Ansprüchen der philosophischen Tradition ein. Pragmatisten und Historisten werfen dieser Tradition eine Überschätzung der Möglichkeit vor, unserer Erkenntnis und unseren Werten ein rational gewonnenes Fundament zugrunde zu legen. Probleme - so wird auf beiden Seiten gedacht - sind immer bestimmte Probleme, Kontexte sind immer holistisch, Lösungen kreativ und zu neuen Handlungen führend.

Jürgen Habermas, den ich für einen der wenigen Denker halte, die ein Gespür für diese Affinität von Pragmatismus und Historismus haben, spricht zur Kennzeichnung dieser Gemeinsamkeit von einer Radikalisierung der Hegelschen Kant-Kritik (oder Meta-Kritik) und erkennt den epochalen Charakter dieser Umwälzung. ${ }^{11}$ Er hegt aber letztlich den Verdacht, daß beide Schulen in ihrer Kritik am Rationalismus der philosophischen Tradition zu weit gingen bzw. kein Mittel fänden, um auf dem neuen Boden normative Fragen zu beantworten. Das sehe ich, wie sich zeigen wird, anders. Aber mit Habermas glaube ich, daß die Umwälzungen in der zweiten Hälfte des neunzehnten Jahrhunderts, die ich hier mit den Namen „Pragmatismus“ und „Historismus“ bezeichne, das Verhältnis zwischen der Philosophie einerseits und den Natur- sowie Geisteswissenschaften andererseits epochal verändert haben. Wenn alle Problemlösung situativ ist und der Traum von der überzeitlichen rationalen Grundlegung ausgeträumt ist, dann kann die Philosophie auch nicht mehr den Anspruch erheben, metaphysisches oder erkenntnistheoretisches Fundament der Wissenschaften zu sein. Sie kann dann nur noch versuchen, sich selbst als wissenschaftliche Disziplin zu gebärden, wie dies für die frühe Phänomenologie (Husserls „Philosophie als strenge Wissenschaft“) galt und für den Stil und das Programm der analytischen Philosophie weithin typisch ist - oder sie kann sich damit bescheiden, ein herausgehobener Ort der Selbstreflexion zu sein, der Selbstreflexion auf Vorgehen und Resultate der wissenschaftlichen Disziplinen, aber auch der Quellen unseres

11 Vgl. Habermas (1983). 
Erkennen und Wertens in den Erfahrungen des Alltagslebens und in den Erfahrungen, die dieses Alltagsleben überschreiten - den ,außeralltäglichen Erfahrungen“ (Max Weber) oder Erfahrungen der Selbsttranszendenz.

Diese umfangreiche Exposition der Gemeinsamkeiten von Pragmatismus und Historismus und der epochalen Bedeutung des Durchbruchs dieser Denkströmungen war nötig, um den nun folgenden Interpretationen der pragmatistischen Beiträge zu einer Theorie der Geschichte historischen Kontext und intellektuelle Rahmung zu geben. Ohne diese nämlich bleibt, wie sich an einem Großteil der Sekundärliteratur zeigen ließe, nur der Weg einer flachen Reformulierung der entsprechenden Schriften, die damit aber in ihrer Sprengkraft unerkannt bleiben.

Wenn nun also die Pragmatisten sich lange Zeit wenig für die spezifischen Probleme historischer Forschung interessierten, wie könnte ihre Zuwendung zu diesen Fragen aussehen? Wie sollte insbesondere der Pragmatismus von Mead, ein Pragmatismus also, der menschliche Intersubjektivität, körperliche und sprachliche Interaktion, die Herausbildung des Selbst hervorhebt, sich auf die Probleme einer Logik der Geschichtswissenschaft auswirken?

Der erste Befund an dieser Stelle ist ein wenig ironisch. Der früheste ernsthafte Brückenschlag von einem pragmatistischen Verständnis der Intersubjektivität zu den Problemen historischer Interpretation ${ }^{12}$ findet sich nämlich nicht bei Mead, sondern bei Josiah Royce, und unterscheidet sich zudem stark von Meads eigenen späteren Versuchen in dieser Richtung. Royce, der einer von Meads akademischen Lehrern im Harvard der 1880er Jahre gewesen war ${ }^{13}$, war ein jahrzehntelanger Freund der beiden Gründerfiguren des Pragmatismus, Peirce und James. Aber bei allen seinen Verdiensten und Leistungen scheint es mir so, als habe er die entscheidende Einsicht, aufgrund derer ich ihn zu den wichtigsten Vertretern des Pragmatismus (und nicht zu den Neoidealisten) rechnen würde, erst nach James' Tod 1910 gehabt. Er erkannte, was die größte Schwäche von James’ epochemachenden Beiträgen zur Philosophie und Psychologie der Religion war, und daß eben diese Schwäche mit Hilfe der Semiotik von Peirce behoben werden könnte. Konkreter gesagt: So großartig James’ Analysen religiöser Erfahrungen waren,

12 Ich stimme zwar Colapietros (2004; 2006) These zu, daß wir Peirce selbst durchaus als Historisten interpretieren sollten, sehe aber doch einen Abstand zwischen dieser Behauptung und der weitergehenden These, daß Peirce grundlegend über die Probleme der historischen Forschung nachgedacht habe. Diese weitergehende Behauptung stellt auf der Grundlage von Peirce' Interesse an der Forschung zur antiken griechischen Philosophie Maria Liatsi (2006) auf. Die Auseinandersetzung mit diesen Schriften kann ich im vorliegenden Zusammenhang nicht leisten; ich gehe deshalb hier auf Peirce und sein Verhältnis zum Historismus bzw. zur Geschichtswissenschaft nicht näher ein.

13 Vgl. Mead (1917). 
es fehlten ihm weitgehend die Mittel, außerdem zu analysieren, wie Menschen, denen eine intensive religiöse Erfahrung zuteil wurde, diese Erfahrung in die Interpretationsmuster ihres Alltagslebens integrieren. Es sah bei James, zumindest in den „Varieties“ von 1902, so aus, als wüchse die subjektive Interpretation einer intensiven individuellen Erfahrung einfach organisch aus dieser Erfahrung heraus, als sei sie lediglich eine Emanation dieser Erfahrung. Von einer Sensibilität für die Schwierigkeiten der Artikulation von Erfahrungen kann nur in einigen wenigen Passagen von James’ Religions-Buch gesprochen werden, etwa dort, wo er die Paradoxie behandelt, daß mystische Erfahrungen sowohl „ineffable“ wie überreich an noetischem Gehalt erscheinen. In diesem Sinne kann man von einem hermeneutischen Defizit der Jamesschen Phänomenologie sprechen. ${ }^{14}$ Die große Einsicht von Royce in seinem späten Meisterwerk The Problem of Christianity von 1913 war es nun, dieses Defizit auf ein mangelndes Interesse von James an der Semiotik von Peirce zurückzuführen. Auch Peirce selbst hatte seine Semiotik nicht fruchtbar auf James’ Religionsphänomenologie bezogen. Aber Royce sah nun, wie revolutionär die Implikationen dieser Semiotik für unser Verständnis von „self“ und „community“ waren.

Wenn nämlich unser Verhältnis zur Welt, wie es Peirce' Semiotik besagt, durch und durch zeichenvermittelt ist, dann haben die Subjekte auch zu sich selbst keinen rein intuitiven, sondern nur einen zeichenvermittelten kognitiven Zugang. Dann ist ihr Verständnis anderer zeichenvermittelt und nicht etwa eine analogische Erschließung des Anderen auf Grundlage eines ursprünglich intuitiven Selbstzugangs. Dann sind „Gemeinschaften“ nicht Aggregationen von autonomen Individuen, sondern das Ergebnis von Kommunikationsprozessen, in denen sowohl individuelle wie kollektive Identitäten erst gebildet werden. Für Peirce und Royce ist der entscheidende Punkt, daß wir das dualistische kantianische Schema von „perception“ und „conception“, sinnlicher Wahrnehmung und begrifflicher Schematisierung, überwinden und durch ein triadisches Schema ersetzen müssen: Jemand interpretiert etwas für jemanden. Dies, so Royce ausdrücklich, gelte auch für Prozesse der Selbstreflexion, in denen eine Person sich selbst für sich selbst interpretiert. Interpretation wird so zur zentralen Kategorie für alles Menschliche. Jede neue Interpretation nimmt selbst die Gestalt eines neuen Zeichens an und wird zum Gegenstand neuer Akte der Interpretation. „Der Wille zur Interpretation“ heißt eines der Schlüsselkapitel in Royce’ Buch, gewiß eine Anspielung auf Schopenhauer und auf den „Willen zur Macht“ Nietzsches, mit denen Royce sich auseinandergesetzt hatte.

14 Vgl. Joas (1997), 67-68. 
Ein zeitliches Ende dieses Interpretationsgeschehens ist nicht vorstellbar. In mehreren Kapiteln, und über Peirce hinausgehend, zieht Royce Schlußfolgerungen aus seiner Theorie der Interpretation für unser Verständnis der Zeit. Die Zeit, schreibt er, „erscheint in neuem Licht durch den Bezug zum Prozeß der Interpretation [...] die Gegenwart interpretiert potentiell die Vergangenheit gegenüber der Zukunft, und so geht dies ad infinitum weiter“15. In dieser Perspektive werden das Gedächtnis und die Rolle expliziter Rekonstruktionen der Vergangenheit zentral, ebenso die Hoffnung und die Rolle antizipierter Zukünfte, und beides gilt für das individuelle Selbst und für die Gemeinschaft. Royce distanziert sich hier von James und wirft ihm eine übertriebene Betonung derjenigen Phänomene vor, in denen eine Verschmelzungserfahrung und ein mystischer Verlust der Persönlichkeit stattfinde. Ohne die Existenz solcher Phänomene zu leugnen, hebt Royce stärker die Rolle des Willens auch in den Prozessen der Herausbildung des Selbst hervor, da wir doch Entscheidungen darüber träfen, ,wie viel von der Vergangenheit und von der Zukunft idealerweise Eingang in mein Leben finden und zum Wert dieses Lebens beitragen soll“16. An dieser Stelle wird deutlich, daß es Royce um zwei Dimensionen im Verständnis von Selbst und Gemeinschaft zugleich ging: die temporale und die ideale Dimension. Ein Selbst, schreibt er, ist nicht „ein bloßes Datum in der Gegenwart, oder eine Sammlung solcher Data, sondern beruht auf einer Interpretation des Sinns, der Tendenz, der Kohärenz und des Wertes eines Lebens, zu dem das Gedächtnis seiner eigenen Vergangenheit gehört.“17 „Ein Selbst ist, eben in seinem Kern, ein Wesen mit einer Vergangenheit. Man muß nach rückwärts im Strom der Zeit blicken, um das Selbst oder seinen Schatten zu sehen, wie es sich mit dem Strom bewegt oder in den Strömungen zwischen den Ufern seines Wasserlaufs herumtaumelt oder voller Anstrengung vorwärtsstrebt bei der Verfolgung seines eigenen, von ihm gewählten Gutes. “18

Zeitlichkeit, so würde ich diesen Gedanken benennen, ist zwar eine konstitutive Dimension des Selbstseins, aber es ist notwendig eine im Licht von Idealen interpretierte Zeitlichkeit. Ohne das, was Royce „ideal interpretation“ nennt, d. h. Interpretation im Licht von Idealen, wäre auch unser interpretiertes Leben „a mere flight of ideas, or a meaningless flow of feelings“19.

15 Royce (2001), 288.

16 Ebd., 253.

17 Ebd., 245.

18 Ebd., 244.

19 Ebd., 253. 
Alles bisher Gesagte gilt auch für Gemeinschaften und deren Selbstverständigung. Gemeinschaften produzieren Ideale - und Ideale produzieren Gemeinschaften. Am stärksten interessiert sich Royce dabei für das Christentum, dem das entsprechende Buch gewidmet ist. Er argumentiert wie einige deutsche protestantische Theologen der Zeit, namentlich Wilhelm Bousset und Ernst Troeltsch, daß wir den christlichen Glauben selbst nicht einfach als Ausfluß von Jesu Lehren auffassen dürften, sondern als das „Produkt des Lebens der frühesten christlichen Gemeinde selbst. Einmal etabliert und dann von Generation zu Generation übermittelt, war dieser Glaube aber unablässig mitschöpferisch bei der Entstehung von Gemeinschaften in jedem folgenden Zeitalter“20. Für diese Gemeinschaften sind - wie die aktive Erinnerung für Individuen - Akte des Gedenkens konstitutiv: Hoc facite in meam commemorationem! Tut dies zu meinem Gedächtnis! Ebenso muß die Zukunft der Gemeinschaft mit der Frage des Individuums nach seiner Zukunft vermittelt werden, auch seiner möglichen Zukunft nach dem physischen Tod. Royce preist die Deutung des Auferstehungsgeschehens durch Paulus als geniale Lösung dieses Problems.

Bei Mead, dem Säkularisten, wären diese Ausführungen nicht vorstellbar, aber ansonsten ließe sich sagen, daß Royce' Philosophie hier alles das entwickelt, was man sich von Meads Ausgangspunkt her erwarten könnte. Es ist deshalb überraschend, daß sich Mead in seinen Arbeiten nie auf Royce' großes Buch von 1913 bezieht. Dasselbe gilt übrigens für John Dewey und für die amerikanischen Historiker. Bei den Historikern kommen Peirce' Semiotik und Royce' Philosophie der Interpretation nicht vor. Eine Erklärung für diesen Befund habe ich nicht.

Uns heutigen Lesern sticht ins Auge, daß Royce' Theorie dem so nahe kommt, was wir von Mead erwarten würden, daß aber Mead selbst für seine Philosophie der Zeit einen ganz anderen Ausgangspunkt wählt. Seine „Philosophy of the Present“ bezieht sich vor allem auf die Naturwissenschaften, nämlich auf die Debatten über Naturgeschichte und die Darwinsche Evolutionstheorie einerseits, auf das neue Verständnis der Zeit in der Physik nach Einstein andererseits. Außerdem profiliert Mead seine Philosophie der Zeit als radikalen „Präsentismus“, als Ausdruck eines Bewußtseins, daß unsere Existenz riskant und endlich in der Zeit ist.

Der Titel von Meads Philosophy of the Present ist bekanntlich ein Wortspiel. ${ }^{21}$ Die unschuldige Bedeutung ist einfach der Bezug auf die Lage der Philosophie

20 Ebd., 256-257.

21 Im folgenden stütze ich mich auf Passagen aus Kapitel 8 (,Zeitlichkeit und Intersubjektivität“) meines Buches über Mead (Joas 1980, 164-194). Vgl. außerdem zum Thema Maines et al. (1983), Baert (1989), Leclerc-Olive (2012), García Ruiz (2013). 
um 1930. Der tiefere Sinn liegt in dem eben erwähnten „Präsentismus“, d. h. im Widerstand gegen eine Philosophie der Vergangenheit, die die Gegenwart nur als Wirkung vergangener Ursachen denkt, und gegen eine Philosophie der Zukunft, die die Gegenwart nur als Stufe in einem Prozeß sehen kann, der durch sie hindurch geht, und dessen utopisches oder apokalyptisches Ziel feststeht. Mead und die anderen Pragmatisten hatten den Darwinismus als radikale Temporalisierung der Natur und Grundlage für ein anti-deterministisches Weltbild empfunden; sie sahen sich aber jetzt durch eine neue Revolutionierung des wissenschaftlichen Weltbilds bedroht. Zwar erschien ihnen Einsteins Relativitätstheorie als Bestätigung ihrer philosophischen Arbeit an einer Überwindung der Vorstellung linearer Zeit, sie waren jetzt aber damit konfrontiert, daß die dominanten philosophischen Interpretationen von Einsteins Innovation durch den Begriff einer vierdimensionalen Raum-Zeit-Welt wieder in ein Denken zurückfielen, demzufolge die Zeitlichkeit ein bloß subjektives Phänomen sei. Gegen diese philosophischen Deutungen stützte sich Mead auf ein pragmatistisches Verständnis des praktischen Charakters der Messung von Zeit. „Gleichzeitigkeit“ war nach Einstein nicht mehr wie im Newtonschen Weltbild als rein objektive Koinzidenz zweier Bewegungsvorgänge in einem Zeitpunkt, unabhängig von jeder Beobachterperspektive, denkbar. Der „Zeitpunkt“ erwies sich vielmehr als jenes endliche Zeitintervall, in dem wahrnehmbare Signale vom Ort des Geschehens aus im Punkt des Beobachters zusammentreffen konnten. Durch die Einsicht in die nicht unendliche, sondern ebenfalls begrenzte Ausbreitungsgeschwindigkeit des Lichts zwang sich damit die Erkenntnis auf, daß der Begriff der „Gleichzeitigkeit“ nicht abgelöst vom Abstand des Beobachters von den Ereignissen und von der Bewegungsrichtung und -geschwindigkeit des Beobachters gefaßt werden könne. Damit aber war mehr geschehen als nur eine Klärung meßpraktischer Fragen. Es war nun nämlich unabweisbar geworden für jede wissenschaftliche Aussage über die Welt bewegter Körper, die Körperlichkeit des Untersuchenden selbst objektivierend miteinzubeziehen. Jede Messung beruht auf praktischen Handlungen erkennender Subjekte; jede Erkenntnis muß selbstreflexiv den Ort und die Perspektive des erkennenden Subjekts mit einbeziehen. Mead fand Bestätigung für sein Bestreben in den Arbeiten Alfred Whiteheads, dem er den Versuch attestierte „Bewegung und Wandel in einem relativistischen Universum zu retten“22. Zentral wurde für ihn dabei der Whiteheadsche Gedanke einer objektiven Realität der Perspektiven. Perspektiven sind nicht nur räumlich, sondern auch zeitlich. Sie sind nicht nur menschlich, sondern allen Lebewesen und vielleicht sogar allen bewegten Körpern zuzuschreiben. Perspektiven sind nicht nur kognitiv, sondern

22 Mead (1932), 43. 
auch evaluativ. Und Perspektiven sind nicht bloße subjektive Zutat außerhalb des Reichs der Natur, sondern deren Teil. Die Realität besteht aus Perspektiven.

In diesem umfassenden Meadschen Sinn wandeln sich die Perspektiven über Zeit - durch Ereignisse. Für Mead sind diese Ereignisse ebenfalls, auch wenn sie nur dem Subjekt als solche erscheinen, objektive Realitäten, da subjektive Erfahrungen eben immer auch objektive Realitäten sind.

In seinen Berkeley-Vorträgen, die dem Buch Philosophy of the Present zugrunde liegen, und in weiteren teils fragmentarischen, kurz vor seinem Tod verfaßten Manuskripten ${ }^{23}$ hat Mead diese Überlegungen explizit auf die Praxis der Geschichtsschreibung bezogen. Rekonstruktionen von Vergangenheiten werden durch neue Ereignisse ausgelöst. Neue Ereignisse konstituieren neue Vergangenheiten. Eine endgültige Rekonstruktion der Vergangenheit wäre nur möglich, wenn keine neuen Ereignisse mehr auftreten könnten, d. h. im Zustand der Zukunftslosigkeit. Geschichtswissenschaft ist nicht mnemotechnische Übung. „Selbst wenn wir die vergangene Gegenwart in die ihr eigene Realität zurückholen könnten, würde uns das nichts nützen. Wir hätten dann jene Gegenwart und gerade nicht das, was wir mit der Vergangenheit suchen, nämlich die Konstruktion der konditionierenden Eigenart des jetzt gegenwärtigen (Ereignis-)Ablaufs, die uns $\mathrm{zu}$ interpretieren erlaubt, was in der $\mathrm{zu}$ dieser Gegenwart gehörenden Zukunft entsteht. Wenn man sich die Zeit seiner Kindheit in Erinnerung ruft, kann man sich nicht - in völliger Beziehungslosigkeit zu dem, was man geworden ist - so in sie hineinversetzen, wie man damals war; und selbst wenn man das könnte, wenn man also die Erfahrungen, wie sie damals waren, reproduzieren könnte, könnte man sie doch nicht verwenden; denn das hieße ja, daß man sich nicht in der Gegenwart befände, in der man sie allein verwenden könnte. [...] Man kann auch sagen, daß unsere Vergangenheiten immer in der gleichen Weise mental sind wie unsere Zukünfte, die in unseren Vorstellungen vor uns liegen. “24

Mead war - so ließe sich dies zusammenfassen - kein historischer Relativist, sondern ein temporaler Relationist. ${ }^{25}$ Unklar bleibt allerdings, wie sich dieser temporale Relationismus zu Meads normativem Universalismus verhielt.

Bevor ich mich dieser Frage zuwende, möchte ich kurz der Frage nachgehen, was der dritte klassische Pragmatist, der sich den Fragen einer Logik der Geschichtlichkeit zuwandte, beigetragen hat. John Dewey widmete in seiner Logik von 1938 das Kapitel XII, aber auch Teile von Kapitel XXII diesen Fragen, und was er dort sagt, ist weitgehend mit Gedanken identisch, die sich bei Mead finden.

23 Ders. (1929).

24 Ders. (1932), 30-31.

25 Vgl. Natanson (1953). 
Allerdings nennt Dewey Mead hier kein einziges Mal; fairerweise muß man sagen, daß wiederum Meads Philosophie der Zeit gewiß schon unter dem Einfluß von Deweys Buch Experience and Nature von 1925 erarbeitet wurde. Dewey hatte sich schon in einer früheren Phase seiner intellektuellen Entwicklung gezwungen gesehen, seine Auffassungen hinsichtlich der Spezifik historischer Forschung zu rechtfertigen. Als im ersten Jahrzehnt des zwanzigsten Jahrhunderts in amerikanischen philosophischen Kreisen die Kontroverse über das pragmatistische Wahrheitsverständnis besonders hitzig war, wurde die von Dewey vertretene Auffassung, daß es immer gegenwärtige Erfahrung sei, die zu neuer Erkenntnis führe, und daß die Verifikation von Geltungsansprüchen in den praktischen Konsequenzen liege, die diese Erkenntnis in der Zukunft habe, als besonders irreführend im Fall historischer Forschung bezeichnet, da bei dieser doch offensichtlich der Gegenstand in der Vergangenheit und nicht in der Gegenwart liege. Dewey verteidigte sich gegen diese Kritik in mehreren kürzeren Artikeln um 1910 mit dem Argument, seine Kritiker hätten eine für ihn zentrale begriffliche Unterscheidung ignoriert: die Unterscheidung zwischen der „brute existence“ der Wirklichkeit und dem konstruierten Charakter all unserer Aussagen über diese. ${ }^{26}$ In seiner Logik knüpft er hieran an und verwendet das folgende, meines Erachtens höchst plausible Beispiel: „Man nehme einmal an, ich sei im Zweifel darüber, ob ich einen bestimmten Brief zur Post gebracht habe, nachdem ich ihn geschrieben habe. Ich nehme vorläufig an, ich hätte es getan, und vollziehe die Operation, auf die erbetene Antwort zu warten. Die Folge dient dazu, die Richtigkeit meiner Annahme zu bestimmen. Entweder erhalte ich eine Antwort oder nicht. Oder ich befürchte, daß ich den Brief nicht abgeschickt habe. Ich vollziehe die Operation, alle Orte zu durchsuchen, an denen ich ihn möglicherweise abgelegt habe. Wenn ich ihn nicht finde, bin ich noch immer nicht willens, die Idee als schlüssig anzusehen, daß ich ihn abgeschickt habe. Ich schreibe einen weiteren fragenden Brief, um sicher zu sein, ob der erste abgeschickt wurde oder nicht.“27

Robert Westbrook, der große Dewey-Biograph ${ }^{28}$, faßt den Gedanken Deweys so zusammen: „An historical account is a tissue of inferences from traces left by past events. This is the most important thing distinguishing historical narrative from fiction. “29 Mead hätte dem wohl zugestimmt. Dewey macht nur klarer als Mead, daß wir über historische Prozesse nicht sprechen können, ohne eine Gerichtetheit ins Spiel zu bringen. Nur so können wir Sequenzen und Linien

26 Vgl. Dewey (1910).

27 Ders. (1938), 226.

28 Vgl. Westbrook (1991).

29 Ders. (1995), 21. 
behaupten. Deshalb bleibt für Dewey die Narration ein unverzichtbarer Bestandteil historischer Forschung. Obwohl Meads Theorie des Selbst nach einer Fortführung in einer Theorie der Narration ruft, findet sich diese bei ihm praktisch nicht. ${ }^{30}$ Dies scheint mir allerdings nur eine thematische, nicht eine systematische Vernachlässigung zu sein.

Systematisch viel wichtiger ist es, auf die Problemlage zurückzugehen, mit der ich die Interpretation von Meads Zeitphilosophie beendet habe. Ich habe davon gesprochen, daß es unklar geblieben sei, wie Mead sich eine Verknüpfung seiner Philosophie der Zeit und seines moralischen Universalismus vorgestellt habe. So formuliert wird vielleicht nicht klar, was hier auf dem Spiele steht. Es handelt sich dabei um das, was im Historismus als Gefahr des Relativismus oder der Anarchie aufgebrochen und in Diltheys Rede an seinem siebzigsten Geburtstag klassischen Ausdruck gefunden hatte: „Ein scheinbar unversöhnlicher Gegensatz entsteht, wenn das geschichtliche Bewußtsein in seine letzten Konsequenzen verfolgt wird. Die Endlichkeit jeder geschichtlichen Erscheinung, sie sei eine Religion oder ein Ideal oder philosophisches System, sonach die Relativität jeder Art von menschlicher Auffassung des Zusammenhanges der Dinge ist das letzte Wort der historischen Weltanschauung, alles im Prozeß fließend, nichts bleibend. Und dagegen erhebt sich das Bedürfnis des Denkens und das Streben der Philosophie nach einer allgemeingültigen Erkenntnis. Die geschichtliche Weltanschauung ist die Befreierin des menschlichen Geistes von der letzten Kette, die Naturwissenschaft und Philosophie noch nicht zerrissen haben - aber wo sind die Mittel, die Anarchie der Überzeugungen, die hereinzubrechen droht, zu überwinden?“31

Auf Mead und die Pragmatisten bezogen heißt dies: Erlaubt ein radikaler Präsentismus in der Zeitphilosophie noch die Annahme objektiv bewährter Geltungsansprüche im Bereich der Erkenntnis, erst recht aber auch im Bereich der Normen und Werte?

Was Erkenntnis betrifft, scheint Mead keine Zweifel gehabt zu haben, daß die Historizität aller Erkenntnis gleichwohl eine Objektivität kognitiver Geltungsansprüche zuläßt. Seine fast nicht rezipierten Schriften zur Wissenschaftsgeschichte

30 Eine ausgezeichnete Interpretation der Überlegungen Deweys, die hier nur kurz vorgestellt wurden, findet sich jetzt bei Hutt (2013). Er kontrastiert Deweys Position mit dem Neukantianismus Heinrich Rickerts und nimmt Stellung zu den äußerst verschiedenartigen Anknüpfungen an Dewey (von Richard Rorty bis Jeffrey Stout) in der gegenwärtigen amerikanischen Philosophie. Sein Grundgedanke ist dabei der, dass „inquiry“ in Deweys Sinn mehr ist als „conversation“ (Rorty), aber nicht identisch mit methodisch disziplinierter und rein auf Tatsachenzusammenhänge zielender Empirie.

31 Dilthey (1924c). 
belegen dies. ${ }^{32}$ Ich will darauf aber hier nicht näher eingehen. Auf dem Gebiet normativer Geltungsansprüche scheint es mir allerdings offensichtlich, daß sein normativer Bezugspunkt in Gestalt der Idee universaler Rollenübernahme nicht hinreichend mit der Frage nach der Kontingenz der Entstehung dieses normativen Bezugspunkts, ja der Entstehung und Institutionalisierung von Werten überhaupt, vermittelt ist.

Empirisch gesehen, verweist die letztgenannte Frage auf die ganze Geschichte des moralischen Universalismus. Wann entstanden erstmals in der Geschichte der Menschheit religiös- oder philosophisch-ethische Vorstellungen von Menschheit insgesamt und von Menschheit als moralischem Bezugspunkt? Wann wurden diese Vorstellungen rechtlich kodifiziert, z. B. in Gestalt subjektiver Rechte auf nationalstaatlicher Ebene? Wann und wie lassen sie sich über diese Kodifizierung im Recht von Staaten hinaus - überstaatlich - verbürgen? Die Antworten, wiederum empirisch gesehen, liegen in den Forschungen zur Achsenzeit Mitte des letzten vorchristlichen Jahrtausends und zur Geschichte der Menschenrechte, v. a. seit dem achtzehnten Jahrhundert. Theoretisch aber scheint mir klar zu sein, daß die Betonung eines Universalismus der Rollenübernahme bei Mead zwar ein universalistisches Ethos psychologisch verankert, aber dieses nicht selbst in seinen historischen Voraussetzungen und Entstehungsbedingungen historisch durchdenkt. Für eine solche Historisierung reicht es ja nicht hin, den psychologischen Mechanismus der Ausübung eines solchen Ethos aufzuklären. Es geht vielmehr um die Klärung der Herausbildung dieses Ethos. Diese Schwierigkeit konnte verborgen bleiben, solange von ökonomischen und politischen Universalisierungsprozessen entsprechende ethische Wirkungen zumindest als langfristig sich einstellendes Resultat erwartet wurden. Aber diese Hoffnung war Mead wie vielen seiner Zeitgenossen durch den Schock des Ersten Weltkriegs vergangen, und die Zeitphilosophie war ein Ausdruck dieses nicht mehr bestehenden Geschichtsvertrauens. Das Fortschrittsvertrauen war immer schon unbefriedigend gewesen, da sich auch die ,achsenzeitlichen“ religiösen und philosophischen Formen des moralischen Universalismus nicht einfach als Effekte archaischer Großreichsbildungen und die Menschenrechtserklärungen des späten achtzehnten Jahrhundert sich nicht als Ergebnisse einer „bürgerlichen Revolution“ denken ließen. ${ }^{33}$

32 Am wichtigsten Mead (1917).

33 Diese knappen Bemerkungen sollen die Brücke schlagen zu meinen empirisch gerichteten Arbeiten zur Geschichte des moralischen Universalismus, um deren methodologische Reflexion es mir in den vorliegenden Reflexionen über Pragmatismus und Historismus vornehmlich geht. Vgl. Bellah/Joas (2012), Joas (2011; 2014). Der ganze vorliegende Aufsatz stellt in einem 
An dieser Stelle muß sich der Blick darauf richten, wie die durch die Katastrophe des Ersten Weltkriegs erschütterten historischen Gewißheiten im Historismus reflektiert wurden. Für den Historismus noch mehr als für den Pragmatismus, für Deutschland noch mehr als für die USA stellte dieser Krieg einen Epochenbruch dar, einen tödlichen Schlag für jeden Glauben an historische „Entwicklung“. In dieser historischen Lage entwickelte Ernst Troeltsch das, was man mit einem treffenden Ausdruck „existentiellen Historismus“34 genannt hat. Ich sehe sein mehr als tausendseitiges Werk von 1922 Der Historismus und seine Probleme als definitive Kritik an allen teleologischen und evolutionistischen Geschichtsphilosophen, die dem Menschen erlauben, die Abhängigkeit der Geschichte von existentiellen Entscheidungen zu vergessen. Doch - und im Unterschied zu den Stimmen einer jungen Generation der Zeit, die man unter dem Namen ,antihistoristische Revolution“ zusammengefaßt hat - reißt Troeltsch dieses Moment existentieller Entscheidung auch nicht aus der Geschichte heraus, als könnten wir jemals die Geschichte durch eine Art reine Entscheidung überwinden. „Geschichte durch Geschichte überwinden“ war Troeltschs nicht unmittelbar verständliche Formel für seinen „existentiellen Historismus“. Ich habe mich im methodologischen Kapitel meines Buches zur Geschichte der Menschenrechte ${ }^{35}$ darum bemüht, seine Argumentation, deren Faden in der Fülle der Auseinandersetzungen mit konkurrierenden Positionen manchmal nicht leicht zu verfolgen ist, zu systematisieren und aufzuzeigen, wie er vom Ausgangspunkt im „Faktum der Idealbildung“ aus eine konsistente Konzeption der Bedingungen für den universalen Geltungsanspruch historisch entstehender Ideale entwickelt. Diese Darlegungen sollen hier nicht wiederholt werden. Aber der Verweis auf sie erlaubt mir, nun den Konvergenzpunkt von Pragmatismus und Historismus zu bestimmen, von dem zu Beginn die Rede war. Der Pragmatismus begann mit einem Verständnis der Kreativität des menschlichen Handelns und der Zeichenvermitteltheit alles Menschlichen und entwickelte erst spät eine Philosophie der Zeit und des Geschichtlichen, ohne aber jemals die festgehaltenen universalistischen Vorstellungen von Normativität ganz zu historisieren. Der Historismus dagegen begann mit einem radikalen Verständnis von Geschichtlichkeit und Ansätzen zu einem Verständnis von Kreativität und Intersubjektivität. Diese blieben aber verengter und ohne Grundlagen in einer „semiotischen Anthropologie“, einer Lehre vom Menschen als einem Wesen, für das die Zeichenvermitteltheit konstitutiv ist. Zudem wurde

bestimmten Sinn die Ausarbeitung einer Anmerkung meines Buchs zur Geschichte der Menschenrechte dar (Joas 2011, 137, Fn. 17).

34 Spranger 1960, (434).

35 Vgl. Joas (2011), 97-139. 
das Problem eines möglichen Universalitätsanspruchs historisch gezeitigter Ideale anfangs nur zögernd gestellt. Von diesem Ausgangspunkt aus aber erreicht Troeltsch den Punkt, an dem er erkennt, daß für die Lösung des Problems einer kontingenzbewußten, aber universalistischen Philosophie der Geschichte eine Anthropologie benötigt wird, die nicht nur dem schöpferischen Charakter des menschlichen Handelns Rechnung trägt, sondern auch seiner Intersubjektivität - in Troeltschs Sprache „der Erkenntnis des Fremdseelischen“36. Dabei bestreitet auch Troeltsch wie Peirce die Möglichkeit purer Intuition. „Es bleibt immerdar bei der Bindung solchen intuitiven Erkennens an einfache oder abgeleitete sinnliche Vermittlungen“37. In pragmatistischer Sprache heißt das, daß die menschliche Intersubjektivität notwendig zeichenvermittelt ist. Die Ineinanderfügung von semiotischer Anthropologie und affirmativer Genealogie ermöglicht die Synthese von Pragmatismus und Historismus.

Troeltsch sah die Notwendigkeit einer semiotischen Anthropologie; die Arbeiten von Peirce und Mead waren ihm aber offensichtlich völlig unbekannt, was nicht verwundert, weil sie ja an obskuren Orten verstreut publiziert oder noch ganz unveröffentlicht waren, als Troeltsch sein großes Historismus-Buch schrieb $^{38}$. Einen Ersatz dafür gab es bei allem wachsenden Interesse am Verständnis der Zeichen auch in Deutschland (bei Husserl und Heidegger) nicht. Seine Nähe zu diesen Denkern blieb verborgen, weil Troeltsch selbst ja - ich würde sagen: in Ermangelung der Kenntnis von Peirce und Mead - sich einen Fortschritt durch den Rückgang auf Leibniz' Monadenlehre und die Partizipationsphilosophie von Malebranche versprach. Diese boten sich ihm an, weil er Philosophen geistesverwandt fand, denen es darum ging, ihre Einsicht in die menschliche Kreativität nicht „prometheisch“-antireligiös auszulegen, sondern umgekehrt in den schöpferischen Leistungen des Menschen eine Teilhabe am göttlichen Geist, am Geist des Schöpfers, zu sehen.

Man könnte phantasieren, daß es in den späten 1920er Jahren zu einer Begegnung von Troeltsch und Mead hätte kommen können. Die intellektuelle Situation rief geradezu nach einem Dialog von „existentiellem Historismus“ und temporalisiertem, historisch sensibilisiertem Pragmatismus. Ein solcher Dialog fand aber nie statt ${ }^{39}$. Bald nach dem Tode Troeltschs im Jahr 1923 und dem Meads 1931

36 Troeltsch (2008), 991.

37 Ebd., 998.

38 Meines Wissens hat Troeltsch auch die Arbeiten von Josiah Royce nicht zur Kenntnis genommen. Royce wiederum kannte Troeltschs Werk über die Soziallehren (Royce 2001, 135-136) und stützte sich auf dieses.

39 Vielleicht die einzige Ausnahme stellt H. Richard Niebuhr dar, ein Denker, der sich stark sowohl auf Troeltsch wie auf Mead stützte. Ich verzichte hier darauf, von dem hier entwickelten 
wandelte sich das intellektuelle Klima so - durch die Machtübernahme der Nationalsozialisten in Deutschland und die Entthronung des zeitweise hegemonialen Pragmatismus in den USA - daß ein solcher Dialog von niemandem mehr angestrebt wurde.

Auf drei Gebieten, die zum Abschluß nur kurz genannt werden sollen, könnte ein solcher Dialog oder eine sich daraus ergebende Synthese von Pragmatismus und Historismus besonders produktiv sein:

Ein erstes Gebiet zeigt sich, wenn berücksichtigt wird, daß die Pragmatisten, wenn sie sich auf Geschichte bezogen, nicht nur an die Geschichte der Menschheit dachten. Für sie blieb die Geschichte der Menschheit immer Teil der Naturgeschichte, Teil der Geschichte der Evolution der Organismen, aber auch Teil der Geschichte des Kosmos. Das ist heute von höchster Aktualität. Die Fortschritte der Forschung auf dem Gebiet der Evolutionsbiologie führen derzeit zu einer Fülle von Versuchen, menschliches Erkennen und Verhalten, auch Kunst und Religion, im Kontext von „human evolution“ zu studieren. Außerdem machen Umweltgefahren wie die Erderwärmung es unmöglich, von den Auswirkungen der menschlichen Geschichte auf andere Organismen und auf das Klima analytisch abzusehen. Die Einbettung der menschlichen Geschichte in die Naturgeschichte stellt - das hat etwa Michael Hampe hervorgehoben ${ }^{40}$ - eine der großen Leistungen des Pragmatismus dar.

Als zweites Gebiet ist die historische Soziologie zu nennen. In der Regel ohne jeden Bezug zum Pragmatismus haben einige der führenden Sozialwissenschaftler unserer Zeit Überlegungen zu einem ereigniszentrierten Verständnis sozialer Zeitlichkeit entwickelt, die vor allem der Zeitphilosophie Meads verblüffend ähnlich sind. ${ }^{41}$ Gerade sie als empirische Forscher auf dem Gebiet einer historisch orientierten Soziologie verabschieden sich von jeder Vorstellung, es ginge bei dieser Forschung um die Entdeckung von Gesetzen des sozialen Wandels, mit deren Hilfe dieser Wandel im Sinne von „smooth, gradual, predictable, and

Gesichtspunkt aus auf weitere Denker der Zeit wie Paul Tillich und Karl Mannheim einzugehen, die sich jeder in eigener Weise in diese Problemlage verstrickt sahen und nach Auswegen suchten. Wie Friedrich Wilhelm Graf berichtet, erklärte Troeltsch „aus den USA kommenden Kollegen und Studenten, bald einer Ende 1922 an ihn ergangenen Einladung zu einer großen Vortragsreise in die USA Folge zu leisten“ (Graf 2014, 23). Das nährt natürlich meine Phantasie einer Begegnung von Troeltsch mit Dewey und Mead. Während der ersten USA-Reise Troeltschs 1904 scheint es zu einer Begegnung mit William James, auf dessen Arbeit sich Troeltsch sehr stark bezog, nicht gekommen zu sein (Troeltsch 2014, 208-209).

40 Hampe (2006), 141-152.

41 Vgl. Mann (1986), Sewell (2005), Abbott (2001). 
linear processes“42 erklärbar sei. Sie proklamieren, was Historikern und gerade den Vertretern des Historismus, immer deutlich war, nämlich daß „historical temporality is lumpy, uneven, unpredictable and discontinuous“43. Die soziologische Theorie wird deshalb nie kausale Gesetze herausfinden, die für eine Vielzahl angeblich ähnlicher Fälle gelten. Eine vernünftige, von der Geschichtsschreibung belehrte Sozialwissenschaft wird vielmehr nur danach streben, Behauptungen aufzustellen, „deren potentielle Verallgemeinerbarkeit durch ihre Fähigkeit unter Beweis gestellt wird, die vermutliche Entfaltung analoger kausaler Prozesse zu beschreiben“44. Schrittweise Fallgeneralisierung, nicht Gesetzeshypothesen und ihre empirische Überprüfung sind damit das Programm einer an Pragmatismus und Historismus anknüpfenden Sozialwissenschaft.

Schließlich sensibilisiert uns die Verbindung zwischen dem Faktum der Idealbildung und der Einsicht in die Historizität jedes Ideals auch für die spezifischen zeitlichen Qualitäten der Erfahrung von Idealen. Dies ist das dritte zu nennende Gebiet. Wir erfahren die Ideale selbst als zeitlos; unsere Erfahrung der Ideale läßt uns aus dem Fluß profaner Zeit heraustreten. Zwar hält diese Erfahrung vielleicht nur für einen kurzen Moment physikalischer Zeit an; sie ist aber so intensiv mit Sinn durchdrungen, daß sie aus der profanen Zeit herausragt, uns in szenischer Erinnerung bleibt und selbst strukturierend auf die profane Zeit einwirkt. Eine solche sakrale oder sakralisierte Zeit ist die Grundlage für unsere Vorstellungen von „Ewigkeit“. Die radikale Verzeitlichung, die von existentiellem Historismus und temporalisiertem Pragmatismus artikuliert wurde, zerstört keineswegs die Möglichkeit, „Ewigkeit“ zu denken. Sie fordert aber dazu heraus, diese nicht im Sinn einer metaphysischen Alternative zu aller erfahrenen Zeit zu denken, sondern selbst als Dimension unserer Erfahrung. ${ }^{45}$

42 Sewell (2005), 9.

43 Ebd.

44 Ebd., 121.

45 Für wichtige Anregungen auf diesem Gebiet vgl. Taylor (2007), 54 ff. etc.

\section{Literatur}

Abbott, A. (2001), Temporality and Process in Social Life, in: Time Matters. On Theory and Method, Chicago, 209-239.

American Historical Review (1991), AHR Forum: Peter Novick's That Noble Dream: The Objectivity Question and the Future of the Historical Profession, American Historical Review 96.3, 675-708. 
Baert, P. J. N. (1989), The Creation of an Invented Future: An Inquiry into G. H. Mead's

Relatively-Open Future with Special Reference to Sociological Theory, in: International

Philosophical Quarterly 29, 319-338.

Bellah, R. N., u. Joas, H. (Hg.) (2012), The Axial Age and Its Consequences. Cambridge, Mass.

Bernstein, R. (1983), Beyond Objectivism and Relativism. Science, Hermeneutics, and Praxis,

Oxford.

Colapietro, V. (2004), Portrait of an Historicist: An Alternative Reading of Peircean Semiotic, in:

Semiotiche 2, 49-68.

Colapietro, V. (2006), Toward a Pragmatic Conception of Practical Identity. Transactions of the

Charles S. Peirce Society 42, 173-205.

Dewey, J. (1938), Logic. The Theory of Inquiry, New York.

Dewey, J. (1978), A Short Catechism Concerning Truth, in: The Middle Works 1899-1924, Bd. 6, Carbondale, Ill., 3-11.

Dilthey, W. (1924a), Die geistige Welt. Einleitung in die Philosophie des Lebens (= Gesammelte Schriften 5), Leipzig.

Dilthey, W. (1924b), Beiträge zur Lösung der Frage vom Ursprung unseres Glaubens an die

Realität der Außenwelt und seinem Recht [1890], in: ders. (1924a), 90-138.

Dilthey, W. (1924c), Rede zum 70. Geburtstag [1903], in: ders. (1924a), 7-9.

García Ruiz, A. (2013), The Concept of the Present and Historical Experience, in Burke, F. T., u. Skowrónski, K. P. (Hg.), George Herbert Mead in the Twenty-first Century, Lanham, Md., 37-47.

Graf, F. W. (2014), Fachmenschenfreundschaft. Studien zu Troeltsch und Weber, Berlin.

Habermas, J. (1983), Die Philosophie als Platzhalter und Interpret, in: Moralbewußtsein und kommunikatives Handeln, Frankfurt am Main, 9-28.

Hampe, M. (2006), Erkenntnis und Praxis. Zur Philosophie des Pragmatismus, Frankfurt am Main.

Haskell, T. S. (1998), Objectivity is not Neutrality. Explanatory Schemes in History, Baltimore, Md.

Honneth, A., u. Joas, H. (1980), Soziales Handeln und menschliche Natur, Frankfurt am Main.

Hutt, C. (2013), John Dewey and the Ethics of Historical Belief. Religion and the Representation of the Past, Albany, N. Y.

Joas, H. (1980), Praktische Intersubjektivität. Die Entwicklung des Werkes von G. H. Mead, Frankfurt am Main.

Joas, H. (1992), Pragmatismus und Gesellschaftstheorie, Frankfurt am Main.

Joas, H. (1997), Die Entstehung der Werte, Frankfurt am Main.

Joas, H. (2011), Die Sakralität der Person. Eine neue Genealogie der Menschenrechte, Berlin.

Joas, H. (2014), Was ist die Achsenzeit? Eine wissenschaftliche Debatte als Diskurs über Transzendenz, Basel.

Jung, M. (1996), Dilthey, Hamburg.

Kloppenberg, J. (1989), Objectivity and Historicism: A Century of American Historical Writing, in: American Historical Review 94, 1011-1030.

Kloppenberg, J. (2004), Pragmatism and the Practice of History: From Turner and Du Bois to Today, in: Metaphilosophy 35, 202-225.

Leclerc-Olive, M. (2012), Les figures du temps dans la philosophie de George Herbert Mead, in: ders., G. H. Mead, La philosophie du temps en perspective(s), Paris, 9-101.

Liatsi, M. (2006), Interpretation der Antike. Die pragmatistische Methode historischer Forschung, Hildesheim. 
Maines, D. R., et al. (1983), The Sociological Import of G. H. Mead's Theory of the Past, in: American Sociological Review 48, 161-173.

Mann, M. (1986), The Sources of Social Power 1, Cambridge.

Mead, G. H. (1917), Josiah Royce - A Personal Impression, in: International Journal of Ethics 27, 168-170.

Mead, G. H. (1917), Scientific Method and Individual Thinker, in: Dewey, J. (Hg.), Creative Intelligence: Essays in the Pragmatic Attitude, New York, 176-227.

Mead, G. H. (1929), The Nature of the Past, in: Coss, J. (Hg.), Essays in Honor of John Dewey, New York, 235-242.

Mead, G. H. (1932), The Philosophy of the Present, La Salle, Ill.

Mead, G. H. (1938), The Philosophy of the Act, Chicago, Ill.

Natanson, M. (1953), G. H. Mead's Metaphysics of Time, in: Journal of Philosophy 50, 770-782. Novick, P. (1988), That Noble Dream. The „Objectivity Question“ and the American Historical Profession, Cambridge.

Popper, K. R. (1965), Das Elend des Historizismus, Tübingen.

Royce, J. (2001), The Problem of Christianity [1913], Washington, D. C.

Sewell, W. H. (2005), Logics of History. Social Theory and Social Transformation, Chicago, Ill.

Spranger, E. (1960), Das Historismusproblem an der Universität Berlin seit 1900, in: Leussink, H., et al. (Hg.), Studium Berolinense. Aufsätze und Beiträge zu Problemen der Wissenschaft und zur Geschichte der Friedrich-Wilhelms-Universität zu Berlin, Berlin, 425-443.

Taylor, C. (2007), A Secular Age. Cambridge, Mass.

Troeltsch, E. (2008), Der Historismus und seine Probleme [1922] (= Kritische Gesamtausgabe 16.116.2), Berlin.

Troeltsch, E. (2014), Schriften zur Religionswissenschaft und Ethik (= Kritische Gesamtausgabe 6.1-6.2), Berlin.

Westbrook, R. (1991), John Dewey and American Democracy, Ithaca, N. Y.

Westbrook, R. (1995), The Authority of Pragmatism, in: Intellectual History Newsletter 17, 16-24. 